\title{
ETNOLOGÍA DE TRES ESTUDIOS ETNOGRÁFICOS SOBRE LA SALUD Y LA SEXUALIDAD DE LOS(AS) JÓVENES DE LAS CIUDADES DEL DEPARTAMENTO DE SANTANDER (COLOMBIA): MOGOTES, SAN JOAQUÍN Y EL PLAYÓN
}

\author{
Manuel Antonio Velandia Mora \\ Master en Educación, Sociólogo.
}

Doctorando Enfermería y Cultura de los Cuidados, Universidad de Alicante

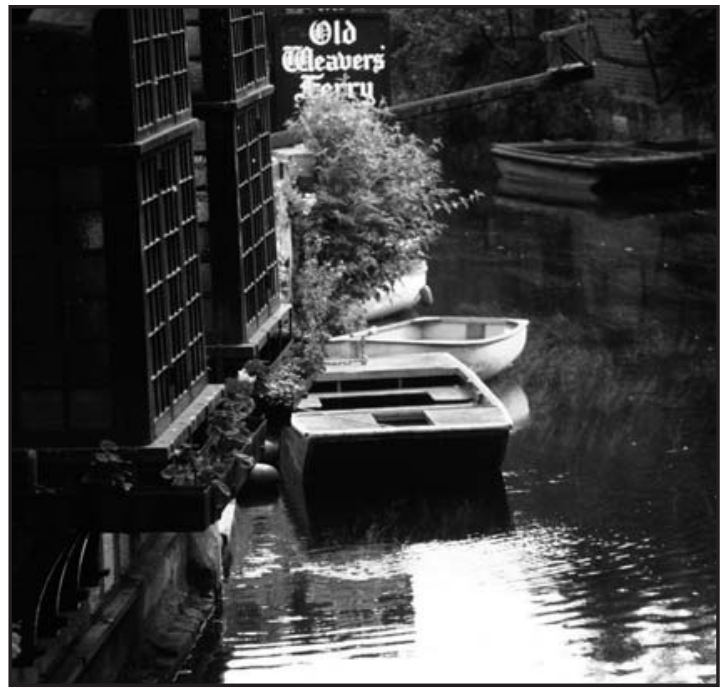

ETHNOLOGY IN THREE ETNOGRAPHIC STUDIES ON HEALTH AND SEXUALITY IN YOUNGSTERS OF DPT.OF SANTANDER (COLOMBIA), MOGOTES, SAN JOAQUÍN AND EL PAYÓN

\section{SUMMARY}

7 he ethnology assembles the results of three researches developed by the author of the text in Mogotes, San Joaquín and El Playón, three municipalities of Santander, Colombia, which population does not exceed 4.000 habitants. In these ethnographic studies in young people, enrolled in school, between the 13 and 19 years, they narrate in groups of discussion, their sexual behaviors, sexual culture and needs in sexual health and reproductive health. The qualitative investigation allowed to obtain a holistic vision, comprehensive and framed in a context of the phe- nomena related with health care, from which was designed an informative and preventive strategy that is implemented nowadays. The socio - critical paradigm grants voice to the student as active subject of the learning process and transforms the communication into an instrument for change. The narrative allows us to interpret the deep complexity of the human nature immersed in situations related to health - disease, due to the fact that they are not linear reducible phenomena. The ethnology made possible to characterize men and women, creating basis for the design of informative and educational strategies, under the statement that young people have the right to have education for the Sexuality, as part of the education for the citizenship; justified, not in religious beliefs, but in results of ethnographies and ethnologies.

Key words: sexual Health, ethnology, ethnography, young, HIV/AIDS

ETNOLOGIA DE TRÊS ESTUDOS ETNOGRÁFICOS SOBRE A SAÚDE E A SEXUALIDADE DOS JOVENS DAS CIDADES DO DEPARTAMENTO DE SANTANDER (COLÔMBIA): MOGOTES, SAN JOAQUÍN Y EL PLAYÓN

\section{RESUMO}

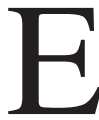
tnologia reúne resultados de três investigações realizadas pelo autor do texto em Mogotes, San Joaquín e o Playón, três municípios de Santander, Colômbia, cuja população não excede os 4.000 habitantes. Estudos etnográficos nos quais jovens escolarizados, entre os 13 e 19 anos, narram em grupos de discussão, 
seus comportamentos sexuais, cultura sexual e necessidades em saúde sexual e saúde reprodutiva. A investigação qualitativa permitiu obter uma visão holística compreensiva e contextualizada dos fenômenos realizados com os cuidados de saúde desde a perspectiva que se desenhou como uma estratégia informativa e preventiva que se implementa atualmente. O paradigma sócio-crítico outorga voz ao estudante como sujeito ativo do processo de aprendizagem e transformação a comunicação em um instrumento para a mudança. A narratividade nos permite interpretar a profunda complexidade da natureza humana imersa em situações relacionadas com a saúde-enfermidade, dado que não são fenômenos lineares redutíveis. A Etnologia possibilitou caracterizar a homens e mulheres, fundamentou o desenho de estratégias informativas e educativas, sob o critério de que os jovens têm direito à Educação para a Sexualidade, como parte da educação para a cidadania, justificadas, não em crenças religiosas, mas em resultados de etnografias e etnologias.

Palavras-chave: Saúde Sexual, Etnologia, Etnografia, jovens, HIV/Sida.

\section{RESUMEN}

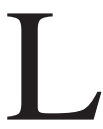

a etnología reúne resultados de tres investigaciones realizadas por el autor del texto en Mogotes, San Joaquín y El Playón, tres municipios de Santander, Colombia, cuya población no excede los 4.000 habitantes. Estudios etnográficos en los que jóvenes, escolarizados, entre los 13 y los 19 años, narran en grupos de discusión, sus comportamientos sexuales, cultura sexual y necesidades en salud sexual y salud reproductiva. La investigación cualitativa permitió obtener una visión holística, comprensiva y contextualizada de los fenómenos relacionados con los cuidados de salud desde la que se diseñó una estrategia informativa y preventiva que se implementa actualmente. El paradigma sociocrítico otorga voz al alumno como sujeto activo del proceso de aprendizaje y trasforma la comunicación en un instrumento para el cambio. La narratividad nos permite interpretar la profunda complejidad de la naturaleza humana inmersa en situaciones relacionadas con la salud- enfermedad, dado que no son fenómenos lineales reductibles. La etnología posibilitó caracterizar a hombres y mujeres, fundamentó el diseño de estrategias informativas y educativas, bajo el criterio de que los jóvenes tienen derecho a la Educación para la Sexualidad, como parte de la educación para la ciudadanía; justificadas, no en creencias religiosas, sino en resultados de etnografías y etnologías.

Palabras clave: Salud sexual, etnología, etnografía, jóvenes, VIH/sida

\section{INTRODUCCIÓN Y OBJETIVOS}

El Departamento de Santander, en Colombia, exigió a las Secretarias Municipales de Salud que como parte del "Plan de Atención Básica "PAB" y sus proyectos de "Salud sexual y reproductiva", realizaran el diseño, implementación y evaluación de una estrategia preventiva en salud sexual y salud reproductiva orientada a los jóvenes entre los 13 y los 19 años. Mogotes, San Joaquín y El Playón son municipios cuya población no excede los 4.000 habitantes, las personas entre los 10 y los 19 años representan el 20,1\% de su población total; las mujeres son el 20,9\% y los hombres el 19,3\%, en relación con este grupo etareo.

Como primera acción de la estrategia preventiva, el consultor-investigador propuso que se implementara en cada uno de los tres municipios, un estudio etnográfico en el que la narración sobre sus comportamientos sexuales, realizada por los(as) jóvenes escolarizados directamente implicados, permitiera conocer sobre su cultura sexual y sus necesidades en el campo de la salud sexual y la salud reproductiva. Los presupuestos del paradigma sociocrítico otorgan voz al alumno como sujeto activo del proceso de aprendizaje y los principios que transforman la comunicación en un instrumento de cambio (Habermas, 1984). La narratividad nos permite interpretar las realidades humanas inmersas en situaciones relacionadas con la salud-enfermedad, dado que no son fenómenos lineales que se puedan interpretar de manera reduccionista (Nicolis, 1999), debido a la profunda complejidad de la naturaleza humana (Morin, 1994). La investigación cualitativa permite obtener una visión holística, comprensiva y contextualizada de 
los fenómenos relacionados con los cuidados de salud (Siles, 2005).

Al ser la primera vez que se implementaba un proyecto de este orden, no se pensó en que fuera necesario un estudio previo al diseño, por sus costos se decidió a partir de los resultados, diseñar una estrategia conjunta aplicable en los tres municipios.

Desde una perspectiva sistémica se consideró necesario hacer conocer la estrategia preventiva a docentes, padres y madres de familia de los jóvenes, a líderes de opinión y al equipo de salud e involucrarlos en los diferentes momentos de la ejecución del proyecto. Los resultados se utilizaron en el diseño de la propuesta preventiva y de los materiales informativos y educativos utilizados en el trabajo directo con los escolares. UNESCO/ONUSIDA (2003), entienden que los estudios de comportamientos sexuales en poblaciones específicas, son pilares fundamentales de la llamada vigilancia epidemiológica de segunda generación de la infección por VIH.

Sobre los jóvenes objeto de estudio no se conocían sus actitudes, comportamientos y prácticas sexuales; no había estudios de prevalencia de VIH/sida/ITS ni sobre sus comportamientos sexuales y no se había realizado hacia ellos ninguna actividad informativa, preventiva o educativa relacionada con el sida, las ITS o el embarazo adolescente. La información que poseían sobre sexualidad, además de lo conocido en la vida cotidiana extraescolar, había sido provista en la única institución educativa de cada municipio y se relacionaba con conocimientos anátomo-fisiológicos. El índice de embarazo adolescente, desde la percepción de la institución educativa y del sector salud, era alto; pero no había estudios que lo confirmaran.

Los/as jóvenes nunca habían sido escuchados/as sobre sus necesidades en educación para la sexualidad, no conocían cómo demandar sus necesidades, ni tampoco se les había ofertado la posibilidad de hacerlo.

Si los jóvenes no son escuchados apropiadamente y no se conoce su cultura sexual, las acciones preventivas y educativas que se diseñen para ellos serán limitadas y no responderán a sus necesidades, así se parta del criterio de que el equipo de salud conoce sus necesidades. Según Leininger, para que haya calidad en los servicios sociosanitarios, los cuidados deben ser culturalmente congruentes ya que para quien se ve imposibilitado a comunicar sus necesidades o demandar apropiadamente sus servicios, se le dificulta la obtención o la prestación de un buen cuidado. Los seres humanos son inseparables de su trasfondo cultural y su estructura social (Siles, Leininger, Velandia).

Objetivo general de la investigación: diseñar, implementar y evaluar una estrategia preventiva en salud sexual y salud reproductiva orientada a los jóvenes hombres y mujeres escolarizados, con edades entre los 13 y los 19 años. Cumplir el objetivo general no era posible sin conocer el trasfondo cultural de la vida sexual de los(as) jóvenes. Para lograrlo se requería conocer sus imaginarios con relación a su sexualidad, la salud y la enfermedad y el embarazo adolescente.

Objetivos específicos: 1. Investigar etnográficamente a la población adolescente escolarizada en la educación media, en cada municipio, para conocer su situación cultural con relación a su salud sexual y la salud reproductiva; 2. Conocer los elementos comunes en los tres estudios etnográficos; 3. Aplicar los resultados al diseño de una estrategia preventiva, para ser aplicada conjuntamente por los municipios; y, 4. Aplicarla y evaluarla.

\section{MATERIAL Y MÉTODO}

Para investigar etnográficamente a esta población se realizaron seis grupos de discusión: uno con hombres y otro con mujeres en cada municipio, y tres "charlas" (grupos de discusión amplios) con participación mixta, para contrastar la información.

Para la recolección de la información se creó un mapa categorial (Mapa Categorial. para el análisis de la información. Cuadro Anexo $\mathrm{N}^{\circ} 1$ ). Las Preguntas Generadoras de dichos grupos se basaron en el análisis sistémico categorial de tres ámbitos de dominio: 1 . Sexualidad; 2 . Salud y enfermedad; y, 3. Bienestar.

Con relación a la "Sexualidad" se analizaron las categorías: 1. Concepto amplio de sexualidad; 2. Ser; 3. Relaciones genitales; 4. Mitos y creencias; y, 5, Embarazo adolescente. Para el binomio categorial "Salud/enfermedad" las subcategorías estudiadas fueron: 1. Embarazo adolescente; 2. 
VIH/Sida e ITS; 3. Calidad de vida. Cabe anotar que la categoría "Embarazo adolescente" es común a los ámbitos de dominio "Sexualidad" y "Salud y enfermedad".

En lo pertinente a la categoría "Concepto amplio de sexualidad" para su análisis se identificó la subcategoría Vivencia de la sexualidad. El "Ser" como categoría, fue contemplado desde tres subcategorías: lo particular, lo social y lo relacional.

En el estudio de las categorías "Relaciones genitales" y "Embarazo adolescente" las subcategorías determinantes fueron: las razones emocionales, los cambios físicos, los cambios emocionales y los cambios relacionales; en la categoría "embarazo", igualmente se tuvieron en cuenta las subcategorías: costos económicos, costos sociales y costos emocionales.

En lo que respecta a la categoría "Mitos y creencias" las subcategorías estudiadas fueron: la Virginidad, la Anticoncepción y la Interrupción del embarazo.

En la categoría "VIH/Sida e ITS" se profundizó en el estudio de las subcategorías: conocimiento, actitud y prevención. Esta última, tiene a su vez tres vice-categorías: saber actuar, saber sentir y saber explicar-se.

La categoría "Calidad de vida" que es común a los ámbitos "Bienestar" y "Salud y enfermedad" se contempla en tres subcategorías: acceso a información apropiada, acceso a medios de prevención y satisfacción de necesidades propias de la edad.

Las Preguntas Generadoras para los grupos de discusión fueron: ¿Cómo viven la sexualidad? ¿Cuál es el lenguaje pertinente a la sexualidad que utilizan?, ¿Cuáles son las razones emocionales en las que se fundamentan sus relaciones sexuales? ¿Cuáles consideran que son los cambios físicos, emocionales y relacionales que ocurren a partir de una relación sexual? ¿Qué piensan de la virginidad? ¿Cuáles son los costos económicos, sociales y emocionales de un embarazo adolescente con relación a la sexualidad, la salud sexual y la salud reproductiva? ¿Qué es el VIH/Sida, cuál es su riesgo y qué hacen para prevenirlo? ¿Qué son las ITS, cuál es su riesgo y qué hacen para prevenirlas?
Criterios de selección de los sujetos participantes: edad, estudiantes de educación media y secundaria, nivel de escolaridad grados séptimo a once, estudiante activo, no lideres estudiantiles, posibilidad de participar de una actividad con dos horas de duración y en horario extraescolar. Para los grupos de discusión, en El Playón: siete hombres entre los 13 y los 17 años y siete mujeres entre los 12 y los 16 años; en Mogotes: ocho hombres entre los 14 y los 17 años y siete mujeres entre los 12 y los 16 años.; en San Joaquín: once hombres entre los 12 y los 16 años y diez mujeres entre los 13 y los 16 años. Doce personas; seis por cada sexo en cada uno de las tres "charlas" o grupos de discusión para la contrastación.

Proceso de captación: presentación previa del proyecto a directivos y docentes. Visita a las aulas de clase, para invitar a los estudiantes a participar voluntariamente en una "charla" sobre las necesidades de los jóvenes del municipio. Se les informó que se podían autonominar para participar de la actividad y que algunos serían seleccionados para trabajar en grupos más pequeños y que, ambos grupos discutirían sobre los mismos temas, se especificó que solamente podían participar entre el 10\% y el $15 \%$ de ellos y ellas, se inscribieron los autonominados; se seleccionaron según criterios preestablecidos para los informantes de los grupos de discusión y la "charla" amplia.

Análisis de la información. Para el análisis de la información se creó un mapa categorial sistémico con cuatro ámbitos de dominio (Mapa categorial epistemológico. Cuadro Anexo $\mathrm{N}^{\circ}$ 2) a saber: 1. Emergencia ${ }^{1} ; 2$. Interdisciplinariedad; 3 . Estudio del Cuidado; y, 4. Cultura.

Con relación a la "Emergencia" se analizaron las subcategorías: 1. Interrelación; 2. Interafectación; y, 3. Interdependencia. Desde la Interdisciplinariedad se tuvieron en cuenta las miradas de: 1. La Enfermería; 2. La Antropología; y, 3. La Sociología.

Para la categoría "Estudio del cuidado" se analizaron tres subcategorías: medio entorno, persona y sociedad. De la conjunción de las subcategorías "medio entorno" y "Persona" surge la vice-categoría "Ámbito de dominio"; y, de la conjunción de

${ }^{\prime}$ La emergencia puede explicarse como una propiedad de todo sistema, del ser humano, dado que este funciona como un todo integrado e integrador y tiene propiedades distintas a las de las partes que lo componen. 
las subcategorías "Persona" y "Sociedad" emerge la vice-categoría: comunidad.

La Cultura, como categoría, se estudia con relación a las mismas tres subcategorías de la categoría "Estudio del cuidado".

La información obtenida en cada uno de los nueve grupos fue grabada en audio, transcrita y analizada desde una perspectiva interdisciplinaria; como emergencia del análisis surgieron dos caracterizaciones, una por sexo.

\section{RESULTADOS}

Caracterización de los hombres: "la sexualidad es un tema urgente, que hay que resolver de alguna manera". Su desinformación lleva a que, una vez enfrentados al tema, no sepan cómo proceder, qué esperar. Consideran que tienen como "ventaja" el hecho de que están en condiciones sociales de exigir y decidir. Lo emocional - afectivo -, erótico y no coital, es un elemento ausente en ellos. Tienden a ser machistas, discriminatorios de las mujeres y homofóbicos.

La sexualidad se restringe al intercambio genital, que es una "necesidad" apremiante, fuera de control, primordial para "hacerse hombre y madurar", y que "es necesario hacerlo pronto". La desinformación en, de y para la sexualidad es amplia, esto conduce a que, una vez enfrentados a la posibilidad de un intercambio genital, un embarazo, el sida u otra ITS, no conozcan cómo proceder, qué esperar, a quién acudir o cuándo hacerlo. Asumen que la masculinidad es una "ventaja" para presionar el intercambio genital, inclusive asumen actitudes y practicas que llegan a la violencia verbal, emocional y física. La efectividad, la ternura, las demostraciones de cariño son poco frecuentes en las relaciones, incluso consideran que "tanto sentimiento es falta de hombría". No hacen referencia a lo erótico, pues "lo importante es ir al grano". Hablan tranquilamente de la masturbación que es "una manera de calmarse si se está arrecho" Prefieren no hablar sobre la planificación de sus intercambios sexuales. El sida no les preocupa porque "en el pueblo solo hay una pareja que lo tiene y no nos metemos con ellos". No usan condones porque "en la droguería (farmacia) los venden, pero allí todo se sabe y los padres se enteran".

$\mathrm{Su}$ principal meta es viajar, la segunda, tener una economía propia, y la tercera, educarse. No ven en la formación académica el cumplimiento de sus metas, sino "una obligación para cumplir con los padres". En sus metas no se encuentra tener hijos, son "un inconveniente y el resultado de un acto no planeado". Ven en los miembros de la policía un fuerte rival para la consecución de una pareja.

Caracterización de las mujeres: tienen un gran desconocimiento sobre sexualidad, cuerpo, erotismo y genitalidad, con un mayor grado de desconocimiento que los hombres. Su sexualidad es un tema tabú, difuso, del que poco desean hablar por considerarlo propio de la intimidad. En caso de autorizarse a una relación sexual genital, ésta "es una manera de amarrar a la pareja". Reciben el peso emocional, cultural y social de administrar su vida sexual, genital y afectiva. Inician su vida sexual genital muy temprano, más por presión de los hombres que por una necesidad particular. Consideran que "el amor y el sexo" (la genitalidad) son una misma cosa, "ellos necesitan que uno les de pruebas de amor". "Con las relaciones sexuales uno se "desarrolla" como mujer"; "es un escalón a la madurez física y emocional”. El erotismo, más presente en ellas que en sus compañeros, es tímido y fruto de la presión del hombre que "desea satisfacer sus necesidades"; no lo reconocen como una posibilidad del Ser o como una alternativa sexual, aun cuando lo relacionan con "gozarse con la pareja”. Asumen que si únicamente hay juego erótico "no pasó nada". No referencian prácticas oralgenitales, ven la masturbación como "algo de ellos y no de mujeres"; aun cuando si saben que "otras niñas se tocan ahî"; prefieren no hablar de este tema pues se asume como "algo sucio, que es pecado". La búsqueda del placer es una meta de los hombres que ellas se ven obligadas a satisfacer, "así uno no quiera, si a él le provoca, si el tiene ganas, a uno le toca, porque si no lo hace ellos creen que ya no los quiere".

Las relaciones de noviazgo se centran en la lucha del hombre por alcanzar su genitalidad y la de ellas en "hacerse las difíciles"; "serlo es sinónimo de entereza, fortaleza y control emocional". "El embarazo no es algo que uno desee, es algo que resulta, porque uno nunca piensa que le va a pasar", "es fruto del amor, no conozco a ninguna que se haya embarazado de alguien que no ame, a no ser que sea violación”. Las relaciones sexuales 


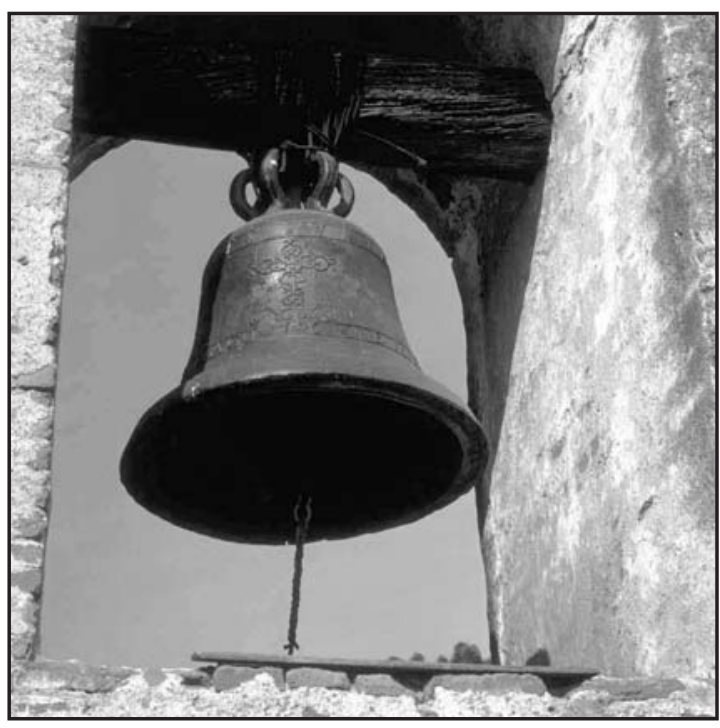

son legítimas y vinculadas al amor, "no a la pasión" y "son propias del noviazgo" (de la Cuesta, 2002), así sus padres no estén de acuerdo, "ellos se olvidan que también fueron jóvenes"; "dicen que no pero se ponen felices cuando son abuelos". Sus relaciones genitales las prefieren lejos de sus casas, en lugares poco comunes como los cultivos ubicados en las afueras del pueblo, el río, el cementerio, y la iglesia. Su sexualidad es "patrimonio familiar" en el sentido de que, muy a su pesar, "lo que una mujer hace con su cuerpo compete a sus padres, mientras que en los hombres no". Ellas reciben el peso de administrar socialmente la virginidad, la "decencia", el embarazo, la maternidad, el desprecio, el aborto y el señalamiento. El afecto o más concretamente "el amor es una necesidad". No llegan a una relación sexual en igualdad de condiciones y oportunidades que los hombres. Son un poco más "exactas" y menos vergonzosas que sus compañeros para referirse a la terminología sexual relacionada con la anatomía tanto femenina como masculina. Su principal meta es conformar una familia, a costa de lo que sea, dada la imposibilidad de viajar, o tener otras alternativas; ven en los miembros de la policía una buena alternativa como pareja y para salir del pueblo, su segunda necesidad; en tercera instancia, quieren educarse o tener una economía propia.

Aspectos comunes en hombres y mujeres: La desinformación sobre el tema de sexualidad y a quién acudir para que les proporcione información confiable, son común denominador en hombres y mujeres. Los padres y maestros no se consideran referentes apropiados, cuando son consultados reprimen, "ellos no oyen, solo les interesa lo que ellos piensan y no lo que nosotros sentimos". Maturana afirma que el fenómeno de la comunicación no depende tanto de lo que se entrega como de lo que pasa con el que recibe. Así, cuando un/a joven y un padre, maestro profesional de la salud escuchan, generan un mundo interpretativo; para Etcheverría (1996), decimos lo que decimos y los/as demás escuchan lo que escuchan; decir y escuchar son fenómenos diferentes. Este aspecto toma especial relevancia en la medida en que asumimos que lo que decimos es lo que los otros escuchan y lo que escuchamos es lo que los/as demás han dicho, si lográramos trascender esta idea y nos tomáramos el tiempo para verificar si estos sentidos concuerdan, estaríamos seguros de que realmente estamos comun-unicando. Los/as jóvenes prefieren usar eufemismos, el sarcasmo y el humor como alternativas comunicativas, para hablar de sexualidad, embarazo, aborto y el uso de condones, que son temas tabú, pero de innegable existencia; lo cual supone cierto acuerdo social al respecto. Los encuentros sexuales genitales son casuales, fruto de la oportunidad o la sumisión en ellas y la presión afectiva de ellos, y casi nunca resultado del acuerdo o la planeación.

Desinformación sobre temas de salud como las ITS incluyendo el sida. No conocen sus derechos sexuales, o sus derechos reproductivos. Temen acudir al hospital (que igualmente es centro de salud) por el temor de que su información privada se haga pública. Les llama la atención el tema de la violencia intrafamiliar, en especial la generada desde las mujeres, pero ven normal la violencia de genero ejercida por los hombres, incluso contemplan los celos y la violencia física como "pruebas de amor".

\section{CONCLUSIONES Y DISCUSIÓN}

En el cotidiano aprendemos los conceptos normativos, básicamente en la familia, la escuela y la iglesia; aquí también aprendemos las organizaciones y las instituciones como la familia, el sistema político, el mercado laboral, el sistema educativo y la política, en consecuencia también aprendemos 
las relaciones de poder, de dominación-dependencia, de inequidad, el discurso y las experiencias aceptadas o no en torno a las sexualidades; en la vida cotidiana aprendemos las identidades, las subjetivamos y construimos las subjetividades masculinas y femeninas (Velandia, 2003a). Con todos estos elementos explicamos y representamos el cuerpo, el género, las orientaciones sexuales, las expresiones comportamentales sexuales, en fin, la sexualidad, el "deber ser" tanto para unos como para las otras.

Aun cuando el diagnóstico se centró en los y las jóvenes escolarizados, las políticas locales deben igualmente reflejar las necesidades especiales de los y las jóvenes desescolarizados/as y de aquellos/as que han abandonado la escuela. Hacer conocer los resultados de la caracterización, a los padres y madres, autoridades civiles en especial de la educación y la salud, a los maestros y maestras y al equipo de salud posibilitó que comprendieran la necesidad de la educación para la sexualidad. Aun cuando los padres se mostraron interesados en conocer la identidad de los informantes, conservar su anonimato es conveniente si se desea que los jóvenes vean a los agentes de prevención como sus aliados.

Los resultados iniciales de cada estudio se implementaron en la elaboración de los contenidos y materiales de soporte de las estrategias informativas y educativas orientadas a las diferentes comunidades educativas: escolares (estudiantes y docentes); padres y madres de familia, líderes de opinión y equipo de salud. Las evaluaciones realizadas por los jóvenes destacaron el conocimiento que los agentes de prevención tenían sobre su realidad, el uso de semánticas que les eran comunes y el reconocimiento de su identidad juvenil. Se insistió para que el diseño de las estrategias preventivas informativas tuvieran en cuenta, como eje del proceso informativo y educativo para al prevención, las condiciones y necesidades emocionales y afectivas de los y las participantes, recalcando que la decisión sobre cualquier práctica sexual genital es necesariamente emocional.

\section{Recomendaciones}

- Los jóvenes tiene derecho a Educación para la Sexualidad; debiera hacer parte de la educación para la ciudadanía y justificada, no en creencias religiosas sino en resultados de etnografías y etnologías, teniendo como fundamento las perspectivas de géneros, derechos sexuales y derechos reproductivos, sin olvidar que la sexualidad va mucho más allá de la anatomía y la fisiología de los cuerpos de los hombres y las mujeres. Entendiendo, así mismo, que la sexualidad hace parte de la vida, desde el momento en que un ser nace hasta cuando fallece y que está relacionada con la capacidad de emocionarse y sentir placer, y que además, del aspecto físico genital, involucra todo el cuerpo y los sentimientos.

- Todos los actores y actrices de la comunidad académica deben asumir un rol de importancia, ya que sin tener en cuenta las necesidades de los/as jóvenes, los intereses de madres y padres, y el sentido y la experticia de docentes y equipo de salud, se hace imposible diseñar una estrategia integral y formativa.

- Se deben crear espacios escolares, familiares y sociales para educar sobre la salud y para la sexualidad, sin barreras para las mujeres y con participación activa de los hombres. Los programas deben informar apropiadamente y con regularidad, recuperando para su acción informativa y educativa las semánticas juveniles sobre cuerpo, salud y sexualidad; sus pensamientos, emociones y necesidades deben ser tenidos en cuenta, ya que generan situaciones que afectan su bienestar y calidad de vida.

- Deben crearse las condiciones para evitar cualquier forma de violencia física y emocional hacia las mujeres, en especial en aquellas situaciones en las que los hombres imponen su poder sobre la decisión de ellas, chantajeándolas emocionalmente u obligándolas a tener relaciones sexuales genitales y otras actos eróticos que no son de su deseo o necesidad; pero igualmente, debe tenerse en cuenta el reporte de casos de violencia domestica física y emocional de algunas mujeres hacia estos.

- Es conveniente crear procesos educativos es lo que sea posible experienciar roles y reflexiones acorde al genero; por ejemplo, el 
taller sobre cómo maternar y paternar, en el que se simulaba durante una semana el cuidado de un hijo (representado en un huevo) posibilitó a los jóvenes una visión distinta de su rol como cabezas de familia, madres y padres, aprendiendo a paternar de una manera no machista y sexista y motivando que se interesen por sus proceso emocionales y sus roles de apoyo en el cuidado de los/las hijos(as).

- Se debe recalcar el hecho de que sexo, relaciones sexuales y sexualidad son conceptos y expresiones diferentes del Ser, ello evita limitar la información y la sexualidad de los y las jóvenes a sus actos genitales coitales.

- Es importante facilitar el acceso a elementos protectores del embarazo adolescente y las enfermedades de transmisión sexual, tales como condones, lubricantes y anticonceptivos orales. Se debe acompañar la entrega de insumos con información apropiada sobre su uso correcto y los riesgos que una relación no protegida adecuadamente puede acarrear en sus vidas.

\section{BIBLIOGRAFÍA}

- De la Cuesta, C. (2002). Tomarse el amor en serio: Contexto del embarazo en la adolescencia. Universidad de Antioquia, Medellín, Colombia.

- Etcheverría. Rafael. (3 ${ }^{a}$ Ed.). (1996). Ontología del lenguaje. Dolmen Ediciones. Santiago, Chile.

- Habermas, Jünger (1984) Teoría de la acción comunicativa I: Racionalidad de la acción y racionalización social. Madrid, Taurus.

- Marriner-Tomey, Ann., Raile Alligow, Martha (1994) Modelos y Teorías en Enfermería. Tercera edición. Ed. Mosby/Doyma libros. España. Pág. 424 a 445. Maturana, H. (1997). Emociones y lenguaje en educación y política. Dolmen. Bogotá.

- Morin, Edgar. (1994) Mis demonios. Kairós, Barcelona.

- Siles González, José; Solano Ruiz, María del Carmen; Cibanal Juan, Luis (2005) Cultura de los cuidados. Revista de enfermería y humanidades. $N^{\circ} 18$, Año IX (2. semestre 2005).

- Nicolis, Grégoire (1994). La estructura de lo complejo: en el camino hacia una nueva comprensión de las ciencias / Grégoire Nicolis, Ilya Prigogine; versión española de A. Klein; revisión técnica de José Luis Sánchez Gómez. Alianza, D. L. Madrid.

- Velandia Mora, Manuel Antonio (2003a). Género, masculinidad y salud: Una visión desde los sistemas, las emociones y el lenguaje. Taller sobre Género en: Foro 2003. II Foro en VIH/SIDA/ITS en América Latina y el Caribe. Conocimiento, experiencia y alianza: Estrategia para el futuro. Palacio de las Convenciones de La Habana, Cuba. 7-12 de abril del 2003.
- UNESCO ONUSIDA (2003); División de políticas culturales y diálogo intercultural UNESCO; Comunicación adecuada para el cambio de comportamientos; información, educación, comunicación; El enfoque cultural en la prevención. Colección especial de manuales metodológicos, Manuales metodológicos $\mathrm{N}^{\mathrm{o}} 1$.

- Velandia Mora, Manuel Antonio (2003). Estrategias para la formación en la convivencia democrática. Pontificia Universidad Javeriana. Bogotá.

- Velandia Mora, Manuel Antonio; Bastidas Beltrán; Guillermo Andrés, Luigui Conversa (2005), Módulo 3. Parcela 1. Educación para la Sexualidad. Bogotá: Organización Internacional de Migraciones OIM/ Proyecto Fondo Mundial de Sida, tuberculosis y Malaria. Colombia.

- Velandia Mora, Manuel Antonio (2005). Caracterización sobre los comportamientos sexuales de los y las jóvenes del municipio de El Playón en el Departamento de Santander, Colombia.

- Velandia Mora, Manuel Antonio (2005). Caracterización sobre los comportamientos sexuales de los y las jóvenes del municipio de Mogotes en el Departamento de Santander, Colombia.

- Velandia Mora, Manuel Antonio (2005). Caracterización sobre los comportamientos sexuales de los y las jóvenes del municipio de San Joaquín en el Departamento de Santander, Colombia.

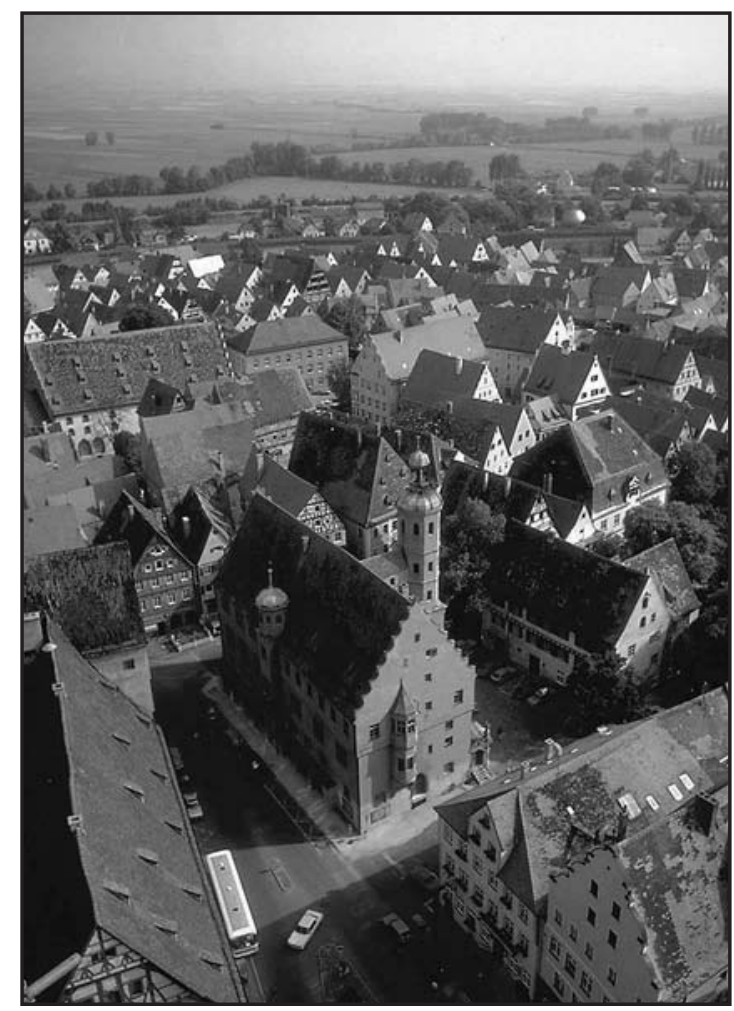


Mapa categorial para el análisis de la información. Cuadro $N^{o} 1$.

\begin{tabular}{|c|c|c|c|}
\hline Ámbito de dominio & Categoría & Subcategori & \\
\hline \multirow{14}{*}{ Sexualidad } & Concepto amplio & \multicolumn{2}{|c|}{ Vivencia de la sexualidad } \\
\hline & \multirow{3}{*}{ - } & \multicolumn{2}{|c|}{ Particular } \\
\hline & & \multicolumn{2}{|l|}{ Social } \\
\hline & & \multicolumn{2}{|l|}{ Relacional } \\
\hline & \multirow{4}{*}{ Relaciones genitales } & \multicolumn{2}{|c|}{ Razones emocionales } \\
\hline & & \multicolumn{2}{|c|}{ Cambios físicos } \\
\hline & & \multicolumn{2}{|c|}{ Cambios emocionales } \\
\hline & & \multicolumn{2}{|c|}{ Cambios relacionales } \\
\hline & \multirow{3}{*}{ Mitos y creencias } & \multicolumn{2}{|l|}{ Virginidad } \\
\hline & & \multicolumn{2}{|c|}{ Anticoncepción } \\
\hline & & \multicolumn{2}{|c|}{ Interrupción del embarazo } \\
\hline & \multirow{6}{*}{$\begin{array}{l}\text { Embarazo } \\
\text { adolescente }\end{array}$} & \multicolumn{2}{|c|}{ Costos económicos } \\
\hline & & \multicolumn{2}{|c|}{ Costos sociales } \\
\hline & & \multicolumn{2}{|c|}{ Costos emocionales } \\
\hline \multirow{9}{*}{ Salud/Enfermedad } & & \multicolumn{2}{|c|}{ Cambios fisicos } \\
\hline & & \multicolumn{2}{|c|}{ Cambios emocionales } \\
\hline & & \multicolumn{2}{|c|}{ Cambios relacionales } \\
\hline & \multirow{5}{*}{ VIH/Sida/ITS } & \multicolumn{2}{|c|}{ Conocimiento } \\
\hline & & \multicolumn{2}{|c|}{ Actitud } \\
\hline & & \multirow{3}{*}{ Prevención } & Saber actuar \\
\hline & & & Saber sentir \\
\hline & & & Saber explicar-se \\
\hline & & \multicolumn{2}{|c|}{$\begin{array}{l}\text { Acceso a información } \\
\text { apropiada }\end{array}$} \\
\hline & Calidad de vida & \multicolumn{2}{|c|}{$\begin{array}{l}\text { Acceso a medios de } \\
\text { prevención }\end{array}$} \\
\hline Bienestar & & \multicolumn{2}{|c|}{$\begin{array}{l}\text { Satisfacción de necesidades } \\
\text { propias de la edad }\end{array}$} \\
\hline
\end{tabular}

Mapa categorial Epistemológico. Cuadro $N^{o} 2$.

\begin{tabular}{|c|c|c|c|}
\hline \multirow{12}{*}{ Análisis sistémico } & \multirow{3}{*}{ Emergencia } & \multicolumn{2}{|c|}{ Interrelación } \\
\hline & & \multicolumn{2}{|c|}{ Interafectación } \\
\hline & & \multicolumn{2}{|c|}{ Interdependencia } \\
\hline & \multirow{3}{*}{ Interdisciplinariedad } & \multicolumn{2}{|c|}{ Enfermería } \\
\hline & & \multicolumn{2}{|c|}{ Antropología } \\
\hline & & \multicolumn{2}{|c|}{ Sociología } \\
\hline & \multirow{3}{*}{ Estudio del Cuidado } & $\begin{array}{l}\text { Medio } \\
\text { entorno }\end{array}$ & $\begin{array}{l}\text { Ámbito de } \\
\text { dominio }\end{array}$ \\
\hline & & Persona & \\
\hline & & Sociedad & Comunidad \\
\hline & \multirow{3}{*}{ Cultura } & \multicolumn{2}{|c|}{ Medio entorno } \\
\hline & & \multicolumn{2}{|l|}{ Persona } \\
\hline & & \multicolumn{2}{|l|}{ Sociedad } \\
\hline
\end{tabular}

